\title{
Dr. Gisela Wild
}

\section{Rechtsanwältin}

\author{
Juristinnen machen Karriere - wir stellen sie vor
}

Das Gespräch führte Birgit Kersten, Vorstand des Regionalgruppenbeirats und Vorsitzende der RG Oldenburg im djb, Rechtsanwältin und vereidigte Buchprüferin, Brake, im November 2010 in Hamburg.

Einer größeren Öffentlichkeit bekannt geworden sind Sie durch Ihre Verfassungsbeschwerde gegen die Volkszählung im Jahr 1983. Wie stehen Sie dem Sammeln von Daten durch die Wirtschaft gegenüber?

Bei Google Street View ist es heute so, dass sich das Unternehmen das Recht nimmt, Daten zu sammeln, die Bürger müssen dagegen Widerspruch einlegen. Google zeichnete bei den Kamerafahrten sogar E-Mails und weitere Daten aus unverschlüsselten WLAN-Netzen auf.

Das Recht auf informationelle Selbstbestimmung, das das Bundesverfassungsgericht im Volkszählungsurteil 1983 als Grundrecht anerkannt hat, beruht auf Artikel 2 Absatz 1 Grundgesetz (allgemeines Persönlichkeitsrecht) in Verbindung mit Artikel 1 Absatz 1 Grundgesetz (Menschenwürde). Es wirkt nicht nur gegenüber dem Staat, sondern in allen Lebensbereichen, somit auch im Bereich der Wirtschaft wie gegenüber Google. Danach hat jeder das Recht zu bestimmen, ob und welche Daten er Dritten gegenüber preisgibt. Voraussetzung für diese Entscheidung ist die Kenntnis der Datensammlung. Heimliche Datenspeicherungen und -sammlungen sind grundsätzlich unzulässig. Kollidiert das Grundrecht des Einzelnen auf informationelle Selbstbestimmung mit anderen Rechten, ist - wie immer in solchen Fällen - im Weg der Güter- und Interessenabwägung zu entscheiden, welchem Recht im konkreten Fall der Vorrang zukommt. Wer selbst seine Daten ungeschützt preisgibt, gibt konkludent sein Einverständnis, sie zu benutzen. Wer das im konkreten Fall nicht will, muss widersprechen.

Das ZDF-Wirtschaftsmagazin WISO hat offengelegt, dass die Einwohnermeldeämter persönliche Daten der Bürger an private Datenhändler verkaufen und dadurch Nebeneinnahmen erzielen. Geht eine größere Gefahr durch das Sammeln von Daten durch den Staat oder die Wirtschaft aus?

Von wem die größere Gefahr ausgeht, von Staat oder Wirtschaft, kann ich nicht beurteilen. Jedenfalls ist beiden Gefahren gleichermaßen zu begegnen.

Benutzer von sozialen Netzwerken wie Facebook, StudiVZ etc. geben sehr viele private Daten von sich preis. Wie ist Ihre Meinung dazu? Wie erklären Sie sich diese Unbefangenheit?

Es ist erschreckend, in welchem Maß Benutzer von Netzwerken persönliche Daten ungeschützt preisgeben. Ich vermute, sie sind von den grandiosen Möglichkeiten der neuen Technik begeistert, haben aber keine Kenntnis von dem, was damit angerichtet werden kann. Deshalb halte ich es für dringend geboten, die Menschen über die Gefahren des Internets aufzuklären.
Gisela Wild wurde am 18. September 1932 in Bad Warmbrunn (Riesengebirge) geboren. Nach der Vertreibung aus Schlesien nach Kriegsende zog die Familie nach Konstanz. 1952 begann Gisela Wild ihr Jurastudium in Freiburg. Ihr Referendariat absolvierte sie in Baden-Württemberg, Köln und Hamburg. 1960 promovierte sie.

1961 wurde Gisela Wild als 16 . Rechtsanwältin in Hamburg zugelassen und fing in der Kanzlei Prof. Bussmann, Dr. Droste an. Sie spezialisierte sich auf gewerblichen Rechtsschutz, Urheber- und Presserecht.

1964/65 lebte Gisela Wild in Paris, wo ihr Sohn geboren wurde.

1970 trat sie als erste Partnerin in die Kanzlei von BerenbergGossler, Frhr. von Gleichenstein in Hamburg ein, die 1990 auf ihre Initiative mit der Kanzlei Wessing in Düsseldorf und Zimmermann in München fusionierte, 2002 erfolgte der internationale Zusammenschluss zur Kanzlei Taylor Wessing

2005 wurde sie von der Bürgerschaft als Hamburgische Verfassungsrichterin gewählt.

Gisela Wild wurde einer breiten Öffentlichkeit durch das sog. Volkszählungsurteil und den Emma-Prozess bekannt.

1996 erhielt Gisela Wild das Bundesverdienstkreuz, 2010 den Maria-Otto-Preis des DAV.

Gisela Wild ist seit 1976 Mitglied des djb, 1977 bis 1979 war sie 2. Vorsitzende, bis 1983 Vorstandsmitglied. Ehrenamtlich engagiert sich Gisela Wild für SterniPark e.V.

Das gilt insbesondere für junge Menschen. An allen Schulen sollte Internetschulung Pflichtfach sein.

Wie sind Sie aufgewachsen? War es für Sie selbstverständlich, Jura zu studieren? Wie sind Sie zum Jurastudium gekommen? Wie war das Studium in den 5oer Jahren? Wie haben Sie gewohnt? Wie war das Verhältnis zu anderen Studenten(innen)? Ich bin die Älteste von vier Kindern. Unsere Heimat war Schlesien. Von dort wurden wir 1946 ausgewiesen; mein Vater befand sich damals in französischer Kriegsgefangenschaft. Nach diversen Zwischenstationen fanden wir eine neue Heimat in Konstanz.

Mein Vater hatte den Ehrgeiz, alle vier Kinder studieren zu lassen, obwohl das für einen Steuerinspektor unmöglich schien und ihm von Seiten seiner Kollegen Spott eintrug. Physik oder Chemie hätte ich gern studiert. Aus finanziellen Gründen kam das jedoch nicht in Frage. So folgte ich dem Wunsch meines Vaters, wie er es nannte, „Kronjuristin“ zu werden, also Jura zu studieren. Ich wehrte mich gegen die hochgesteckten Erwartungen, aber Richterin zu werden, erschien mir als Berufung. 
Um einen finanziellen Grundstock für das Studium zu legen, habe ich erst neun Monate als Arbeiterin bei der Firma Maggi in Singen gearbeitet, in „Suppen II“ zum Stundenlohn von 89 Pfennig, dem damaligen Arbeitslohn von Frauen zwischen 18 und 21 Jahren. Als ich im Wintersemester 1952 in Freiburg mein Jurastudium begann, lag der Frauenanteil unter zehn Prozent. Wir Jurastudentinnen kannten uns natürlich und haben teilweise noch immer Kontakt, paarweise. Denn die Freundschaften, die damals mit Kommilitonen geschlossen wurden, führten zu haltbaren Ehen.

Gewohnt habe ich in einer Mansarde von ca. 12 Quadratmetern mit Kanonenofen und Waschschüssel, Wasser und Toilette über den Flur, zu 30 Mark. Das Budget durfte 100 Mark nicht überschreiten. „Fleißprüfungen“ verschafften Befreiung von den Studiengebühren. In den Semesterferien habe ich gearbeitet, zunächst bei einer Elektrofirma, dann beim Südkurier in Konstanz.

Sie sind Kommentatorin des Urheberrechtskommentars Schricker Loewenheim und haben bereits in Ihrer ersten Stelle 1991 im gewerblichen Rechtsschutz und dem Urheberrecht gearbeitet. War das Zufall oder hatten Sie sich bereits im Studium dafür interessiert? Ist dies das Gebiet, das Ihnen sehr am Herzen liegt und wenn ja, aus welchem Grund? die Zeitschrift STERN wegen sexistischer Titelbilder. Das Verfahren ging verloren, führte aber unter anderem zum Verbot der beruflichen Diskriminierung auf Grund des Geschlechts und der sexuellen Belästigung im Gleichbehandlungsgesetz 1979.

Wie kam es dazu, dass Sie 1964/65 zwei Jahre in Paris gelebt haben? Was haben Sie als Erfahrung aus Frankreich mitgebracht, das Sie nicht missen möchten?

Mein Mann ging 1963 als Korrespondent des SPIEGEL nach Paris. Ich folgte ihm. Unser Sohn wurde in Paris geboren. Während des Aufenthalts studierte ich Europarecht bei Professor Goldmann und übersetzte mit einer französischen Kollegin Europarechtstexte. Darüber habe ich eine Menge französisches Recht gelernt und die französische Kultur liebgewonnen.

In einem Interview mit Ihnen habe ich gelesen, dass Sie Ihre Kanzlei spätestens um 16:30 Uhr verlassen haben, um Ihren Sohn aus dem Kindergarten abzuholen. Die restliche Arbeit haben Sie am späteren Abend erledigt. Damit haben Sie bereits in den 6oer Jahren die lange Anwesenheit, die „.Präsenzkultur" in der Kanzlei aufgebrochen. Das gelingt auch heute nur wenigen Frauen. Und das, obwohl die Anzahl der Anwältinnen geradezu explodiert ist: Sie waren im Jahr Ihrer

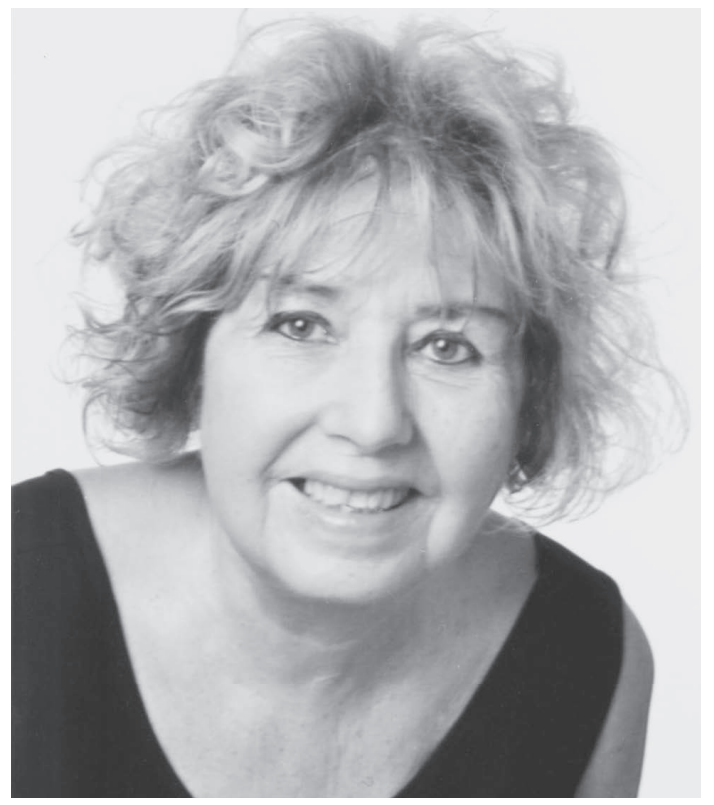

Zum Urheberrecht kam ich über meine erste Stelle als Rechtsanwältin bei Professor Dr. Kurt Bussmann, Dr. Helmut Droste in Hamburg. Die Kanzlei war spezialisiert auf das Gebiet des Gewerblichen Rechtsschutzes und Urheberrechts. Professor Bussmann beriet die Bundesregierung zum Urheberrechtsgesetz, das 1965 in Kraft trat. Seither hatten das Urheberrecht und das Presserecht mein besonderes Engagement, zumal mein Mann als Journalist dazu herausforderte. Über die St. Pauli Nachrichten, die ich 1969 bis 1971 beriet und vertrat, habe ich viel vom Zeitungsgeschäft gelernt. 1972 folgte die Vertretung der von Alice Schwarzer angeführten Klage gegen erstmaligen Zulassung die 16. Anwältin in Hamburg überhaupt. Aktuell sind in Hamburg 2.940 Anwältinnen zugelassen (32\% der 9.248 Mitglieder der Rechtsanwaltskammer in Hamburg). Der Stern titelte gerade „Karriere? Das tue ich mir nicht an!"Warum haben Sie das geschafft? Meinem Eindruck nach sind wir heute kaum einen Schritt weiter. Wie ist das zu erklären?

1965 bekam ich von Dr. Droste die Anfrage, wann ich denn endlich wieder nach Deutschland käme, die Kanzlei brauche mich. Die Frage war, ob ich halbtags oder ganztags arbeiten wolle. Ich sagte natürlich ganztags. Es könnte ihnen so passen, 
mich halbtags zu bezahlen, obwohl klar war, dass sich engagierte Anwaltstätigkeit zeitlich nicht beschränken lässt. Wichtig war, frei über die Zeit bestimmen zu können, dazu gehörte das Abholen vom Kindergarten und der Abend mit dem Kind, soweit nicht besondere Vorkommnisse Improvisationen erforderten. Die Partner der Sozietät hatten dafür volles Verständnis. Das ist auch heute möglich, wenn man will. Leider will man oft nicht - das gilt ebenso für Frauen.

Heike Mundzeck schneidet gerade einen Dokumentarfilm zum Thema „Frühe außerhäusliche Betreuung von Kleinkindern sowie Erziehungspartnerschaft Eltern und Krippe“. Eine Fremdbetreuung für Kinder unter drei Jahren in Krippen war lange Zeit in den alten Bundesländern verpönt. Hier findet ein Wandel in der Akzeptanz statt, Kinder vor dem dritten Lebensjahr in eine Krippe zu geben. Auf einer Tagung habe ich gerade gehört, dass nur für 15 Prozent der Kinder ein Krippenplatz zur Verfügung steht. Da scheint es noch erheblichen Nachholbedarf zu geben. Wie haben Sie damals die Kinderbetreuung geregelt?

Ich hatte ursprünglich ein Kindermädchen, das bei uns wohnte und mir damit große Freiheit gab. Es kostete mich allerdings nahezu das ganze Honorar, das ich damals verdiente.

Ich empfinde es als Fortschritt, das Kind auch schon vor dem dritten Lebensjahr in einer Krippe betreuen zu lassen. Da die wenigsten Familien heute mehrere Kinder haben, die sich wechselseitig sozialisieren, erscheint mir eine gute Krippe mit unterschiedlichen Kindern wichtig für die persönliche Entwicklung.

Was empfehlen Sie Frauen, die gerne in Großkanzleien arbeiten möchten - auch mit Kind?

In einer Großkanzlei zu beginnen und bei Bewährung ein Kind zu bekommen oder auch mehrere. Qualifizierte juristische Mitarbeiterinnen lässt die Kanzlei nicht gern ziehen. Auch als Partnerin ist das möglich. Voraussetzung ist allerdings, dass die Frau überzeugte Rechtsanwältin ist und die Kinderzeit als notwendige Übergangszeit versteht.

Was stellte die größte berufliche Herausforderung für Sie dar? Hat es auch einmal schwierige Situationen in Ihrem Berufsleben gegeben?

Die größte berufliche Herausforderung war, als meine Partnerin Maja Stadler-Euler und ich die Verfassungsbeschwerde gegen das Volkszählungsgesetz erhoben. Schwierig war es auch insoweit, als unsere Partner und auch einige wertvolle Mandanten stark irritiert waren. Ich weiß nicht, was geworden wäre, hätten wir damals verloren.

Worin finden Sie einen Ausgleich zu Ihrem Beruf? Was macht Ihnen besonders viel Freude?

Kultur, Reisen und ehrenamtliches Engagement.

Besonders viel Freude macht mir zur Zeit die Unterstützung von SterniPark e.V., einem gemeinnützigen Träger der freien Jugendhilfe, der rund 1.500 Krippen- und Kindergartenplätze unterhält einschließlich dreier Babyklappen und zweier Mutter/Kind-Einrichtungen zur Hilfe in Not geratener Mütter.

Rechtsanwalt Groenewold und ich beraten SterniPark strategisch. SterniPark steht nicht nur für die Babyklappen, sondern auch für anonyme Geburten, das sind Hilfsangebote für Mütter, die sich in Not befinden und anonym bleiben möchten. Das Konzept wird leider von verschiedenen Seiten angegriffen. Ich verteidige es aus voller Überzeugung.

Der Deutsche Ethikrat hat in seinem Gutachten zu Babyklappen und anonymen Geburten ausgeführt, dass jedes Kind wissen müsse, wo es herkommt. Das geht nicht durch Verbote und Strafen, sondern setzt die Mitwirkung der Mütter voraus. Diese sind durch Hilfsangebote zu gewinnen. SterniPark zeigt, wie es geht. Von fünf Kindern, die 2008 in eine SterniParkBabyklappe gelegt wurden, haben sich vier Mütter gemeldet. Im Streit um die Äußerung des zuständigen Hamburger Senators, „Kinderhandel sei nicht auszuschließen“, veranlasste SterniPark durch den Notar und ehemaligen Bürgermeister von Hamburg, Dr. Henning Voscherau, zu den Fällen eine notarielle Tatsachenbescheinigung, in der die Mütter ihre Geschichten anonymisiert zu Protokoll gaben. Diese Geschichten lassen keinen Leser unberührt. Von den vier Kindern sind inzwischen drei wieder in ihren Familien, ein Kind wurde offen zur Adoption gegeben. Seit elf Jahren bietet SterniPark diese Rettung für Kinder und Hilfe für Mütter in Not.

Jeder neue Kindergarten ist mir eine Freude. Ich habe kein Verständnis für Nachbarn, die sich gegen Kindergärten wehren und mit Klagen verfolgen.

Sie sind bereits seit dem 1. April 1976 Mitglied des djb. Von 1977 bis 1979 waren sie 2. Vorsitzende des djb, bis 1983 Mitglied des erweiterten Bundesvorstands. Welche Themen verbinden Sie mit Ihrer Amtszeit? Wie sind Sie zum djb gekommen und was hält Sie schon so lange im Verband?

Zum Deutschen Juristinnenbund kam ich über Lore Maria Peschel-Gutzeit (zeitweilig Justizsenatorin von Hamburg und Berlin), damals Erste Vorsitzende des Verbandes, die beim Bundesverfassungsgericht ein Gutachten zu meiner Verfassungsbeschwerde zwecks steuerlicher Absetzbarkeit von Kinderbetreuungskosten erstattete. Den djb halte ich für den Verband, der nunmehr schon seit Jahrzehnten unter jeweils hervorragender Führung am qualifiziertesten die Rechte von Frauen und Kindern vertritt, ohne dabei die Interessen der Männer und Väter zu negieren. Das wichtigste Thema meiner Amtszeit war die Neuordnung des Kindschaftsrechts mit der Abschaffung der körperlichen Züchtigung.

Was halten Sie von „der Quote“? Der djb fordert diese schon lange für Frauen in Führungspositionen, insbesondere Aufsichtsräten, deutscher Unternehmen.

Die Quote sollte nur die letzte Maßnahme sein, wenn alle Anstöße zur Veränderung nichts nützen. Das frühere Argument, es gebe keine entsprechend ausgebildeten Frauen, zählt nicht mehr. Aber die Frauen dürfen sich auch nicht verweigern, an der Umgestaltung der Gesellschaft mitzuwirken. Es kommt 
immer wieder vor, dass Frauen höhere Positionen ablehnen, weil ihnen die Belastung zu hoch erscheint. Man muss den Mut haben, selbst etwas verändern zu wollen.

Auch in Ehrenämtern und Gremien sind oft nur wenig Frauen, solange sie kleine Kinder haben. Ich höre jetzt aber neuerdings, dass auch Männer Aufgaben außerhalb der Kanzlei zugunsten der Familie zurückstellen.

Sie haben viele Auszeichnungen erhalten: das Bundesverdienstkreuz, den Liberta Preis, den Maria-Otto-Preis. Sie sind immer selbstbewusst und mutig Ihren eigenen Weg gegangen. Woher beziehen Sie diesen Mut, das Selbstbewusstsein und die Kraft?

Aus der Unterstützung meiner Familie, zunächst meines Vaters, seit Studienzeiten meines Mannes.

Vor der Hochzeit haben mein Mann und ich die Vereinbarung getroffen, dass immer derjenige, der im Beruf das bessere Angebot erhält, dieses auch annimmt und der andere zurücksteckt. Dies hat sich auch eine ganze Weile bewährt. Als ich mich dann hier in Hamburg als Rechtsanwältin etabliert hatte, blieb allerdings nur dieser Standort.

\section{Wie hat sich Ihre Arbeit als Rechtsanwältin im Laufe der Jahre verändert?}

Es ist meines Erachtens ganz wichtig, die passenden Partner im Beruf zu finden und mindestens im eigenen Team ein gutes Klima zu schaffen. Schließlich verbringt man in der Regel über die Hälfte des Lebens im Beruf.

Ich habe von Anfang an im gewerblichen Rechtsschutz gearbeitet. Früher kannten sich die Kollegen bundesweit. Als nach 1990 die überörtlichen Sozietäten entstanden, lag es nahe, über meine guten Kontakte zu Wessing in Düsseldorf und Zimmermann in München den nationalen überörtlichen Zusammenschluss zu begründen.

Rechtsgebiete wie Strafrecht, Familienrecht, Sozialrecht, Verkehrsrecht passen nicht in die heutigen Großkanzleien. In diesen Kanzleien geht es um Fälle, die ein Anwalt allein, selbst mit Hilfe, in der Regel nicht mehr bearbeiten kann. Erforderlich ist ein Team von Spezialisten übergreifender Rechtsgebiete wie Gesellschafts-, Kartell-, Steuerrecht, oft mit Unterspezialisierungen. In der Regel leitet das am konkreten Fall orientierte Teamwork ein erfahrener Partner.

Unsere Hamburger „Betriebsstätte“, wie sie heute heißt, ist über 125 Jahre alt. Bei meinem Eintritt 1970 waren wir fünf Partner, heute zählt Taylor Wessling weltweit über 750 Anwälte. Es ist uns wichtig, von keiner nationalen Gruppe dominiert zu werden.

2002 gab es einen internationalen Schub. Mit den ausländischen Beteiligungen und dem Wettbewerb unter den Großsozietäten wandelten sich auch die Arbeitsbedingungen. Die wirtschaftliche Effizienz wurde zu einem der wesentlichsten Parameter. Die Anwaltszeitschrift Juve berichtet nicht nur über nahezu alle großen Deals und Verfahren, sondern vergleicht auch Umsätze und Effizienzen von Sozietäten und Anwälten. Daraus ergibt sich ein nicht unerheblicher Druck.

Der Präsident der Bundesrechtsanwaltskammer Axel C. Filges, ein Partner von Taylor Wessing, erinnert immer wieder daran, dass Anwälte auch Organe der Rechtspflege sind. Das dürfen wir in der Tat nicht vergessen.

Vielen Dank für das Gespräch, Frau Dr. Wild.

\section{Impressum}

\section{Schriftleitung:}

Anke Gimbal, Rechtsassessorin (V.i.S.d.P.)

Juliane Lindner

\section{Redaktionsanschrift:}

Deutscher Juristinnenbund e. V.

Anklamer Str. 38

10115 Berlin

Telefon: 030 443270-0

Telefax: 030 443270-22

E-Mail: geschaeftsstelle@djb.de

\section{Druck und Verlag:}

Nomos Verlagsgesellschaft mbH \& Co. KG

Waldseestr. 3-5

D-76530 Baden-Baden

Telefon: 07221 2104-0

Telefax: 07221 2104-27

Anzeigenverwaltung und
Anzeigenannahme:
Sales friendly, Verlagsdienstleistungen
Bettina Roos
Siegburgerstr. 123
D-53229 Bonn
Telefon: 0228 97898-o
Telefax: 0228 97898-20
E-Mail: roos@sales-friendly.de

Die Zeitschrift sowie alle in ihr enthaltenen einzelnen Beiträge und Abbildungen sind urheberrechtlich geschützt. Jede Verwertung, die nicht ausdrücklich vom Urheberrechtsgesetz zugelassen ist, bedarf der vorherigen Zustimmung des Verlages.

Namentlich gekennzeichnete Artikel müssen nicht die Meinung des Herausgebers oder der Schriftleitung wiedergeben. Unverlangt eingesendete Manuskripte - für die keine Haftung übernommen wird - gelten als Veröffentli- chungsvorschlag zu den Bedingungen des Verlags. Es werden nur unveröffentlichte Originalarbeiten angenommen. Die Verfasser erklären sich mit einer nicht sinnentstellenden redaktionellen Bearbeitung einverstanden.

\author{
Erscheinungsweise: \\ vierteljährlich \\ Bezugspreis 2011: \\ jährlich 48,-€, Einzelheft 13,-€
}

Alle Preise zzgl. Vertriebs-Direktbeordnungsgebühren inkl. MWSt.;

Bestellungen nehmen entgegen:

Der Buchhandel und der Verlag; Kündigung jeweils drei Monate zum Kalenderjahresende. Zahlungen jeweils im Voraus an: Nomos Verlagsgesellschaft, Postbank Karlsruhe, Konto 7363651 (BLZ 66010075) oder Stadtsparkasse Baden-Baden, Konto 5002266 (BLZ 66250030). 